\title{
Extended Pythagoras Theorem Using Hexagons
}

\section{Luis Teia}

Correspondence: von Karman Institute for Fluid Dynamics, Chaussée de Waterloo 72, 1640 Rhode-Saint-Genèse, Belgium

Received: October 6, 2021

doi:10.5539/jmr.v13n6p46
Accepted: November 13, 2021 Online Published: November 21, 2021

URL: https://doi.org/10.5539/jmr.v13n6p46

\section{Abstract}

This article provides the geometric and algebraic proof of the variant equation of the Pythagorean theorem $x^{2}-x y+$ $y^{2}=z^{2}$. The hypothesis that will be proven is that just as squares govern the original version $x^{2}+y^{2}=z^{2}$, hexagons are found to govern $x^{2}-x y+y^{2}=z^{2}$. Both the special case $x=y$ and general case of $x \neq y$ are examined.

Keywords: Pythagoras, theorem, geometry, trigonometry, extension, hexagon

\section{Introduction}

The equation that governs the Pythagorean theorem $x^{2}+y^{2}=z^{2}$ has been explored in-depth both in its geometric and algebraic significance, with many proofs emerging throughout the years (Euclid et al. 1908, Maor 2007). One variation of this equation is

$$
x^{2}-x y+y^{2}=z^{2}
$$

Considering the deep impact that the Pythagorean theorem has in various branches of science (Rawlins 2000, Parisher \& Rhea 2012, Howard \& Workman 2018, Curtin 2020) - making it a cornerstone in educational mathematics (Canadian Ministry of Education, 2020) - it is expected that the study of this variation of the original equation will unlock further gains in various scientific and educational fields. Cases that are key to understanding the geometric and algebraic behavior of such equations are $x=y$ and $x \neq y$. To begin with, the cosine rule given by $z^{2}=y^{2}+x^{2}-2 x y \cos (\gamma)$ [Pickover, 2012] suggests that just as a reference angle of $\gamma=90$ degrees (where $\cos \left(90^{\circ}\right)=0$ indicates a relation between squares with an external angle of 90 degrees) reducing the said equation to $x^{2}+y^{2}=z^{2}$, a reference angle of $\gamma=60$ degrees (where $\cos \left(60^{\circ}\right)=1 / 2$ indicates a relation between hexagons with an external angle of 60 degrees) reducing the said equation to $x^{2}-x y+y^{2}=z^{2}$.

(a)

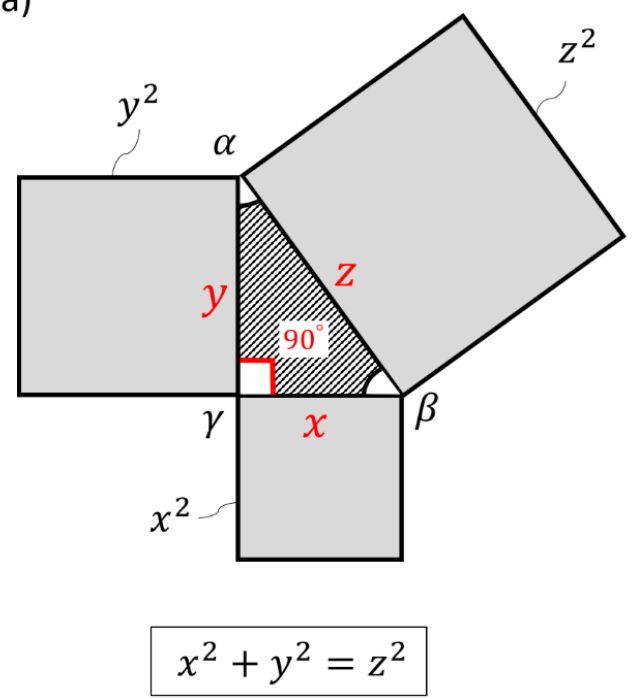

(b)

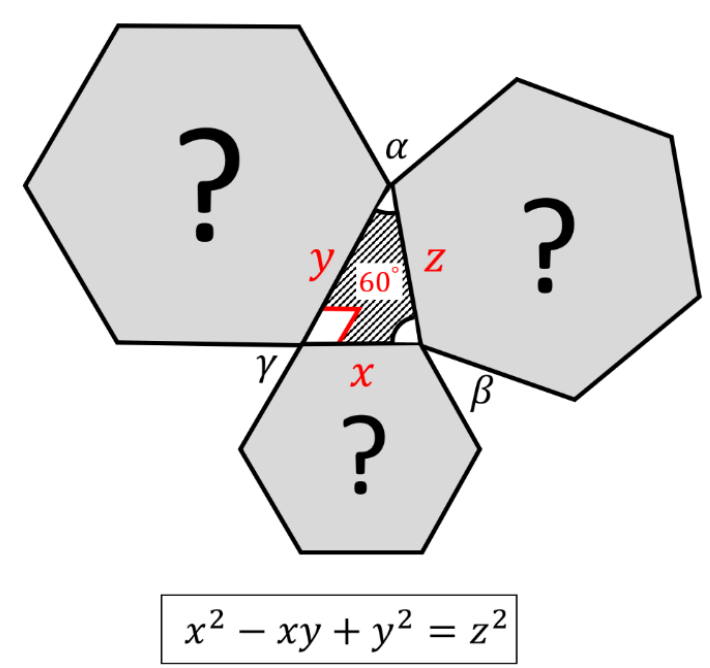

Figure 1. Pythagorean theorems: (a) classical using squares and (b) potentially extended using hexagons 


\section{Hypothesis}

It is the hypothesis of this article that just as squares govern the area relation between the three sides of a right-angled triangle expressed as $x^{2}+y^{2}=z^{2}$, hexagons govern the area relation between the three sides of a 60-degree-reference triangle expressed as $x^{2}-x y+y^{2}=z^{2}$.

\section{Theory}

A theoretical preparation step-by-step of the fundaments that will be required to compose the proof are first presented. This will facilitate the understanding of the proof, in both its geometric and algebraic components. While it is usually a good starting point to examine exceptions in a rule (such as, $x=y$ ), in this case the opposite (i.e., $x \neq y$ ) is found preferable. It is easier to start with the general case $x \neq y$, when interpreting the extension of the Pythagorean theorem to $x^{2}-x y+y^{2}=z^{2}$ using hexagons.

\subsection{General Case $x \neq y$}

Consider the scalene triangle $\mathrm{ABC}$ of reference angle 60 degrees, composed of sides $x=\mathrm{AC}, y=\mathrm{AB}$ and $z=\mathrm{BC}$ (Figure 2). Three regular hexagons are formed around each edge, namely hexagon ACDEFG along the side $x=\mathrm{AC}$ of area $3 \sqrt{3} / 2 x^{2}$, hexagon ABPNML along the side $y=\mathrm{AB}$ of area $3 \sqrt{3} / 2 y^{2}$, and hexagon CBHIJK along the side $z=\mathrm{BC}$ of area $3 \sqrt{3} / 2 z^{2}$. Forming an equilateral triangle $\mathrm{ACO}$ downward along the edge $x=\mathrm{AC}$, and revolving six congruent equilateral triangles (same as ACO) around point O completes the hexagon ACDEFG. In previous proofs, the central theory (Teia, 2015) was used to define relations between right-angle triangles and form triples, in particular by verifying that when these revolves around a central point, their inner sides inherently form an inner (central) square, while at the same time the outer edges form an outer square. Similarly, extrapolating from this theory, it is expected that the same occurs inside hexagon CBHIJK. Mirroring the 60-degree-angled triangle $\mathrm{ABC}$ about the edge $z=\mathrm{BC}$ gives the triangle $\mathrm{BCC}$ ', and revolving six congruent 60-degree-angled triangles BCC' about the centre point O' (that is BCC', HBB', IHH', JII', KJJ' and CKK') forms from the inner edges of the triangles the central hexagon C'B' $\mathrm{H}^{\prime} \mathrm{I}^{\prime} \mathrm{J}$ 'K' of side $y-x$ and area $3 \sqrt{3} / 2(y-x)^{2}$, and from the outer edges of the triangles the hexagon CBHIJK of side $z$ and area $3 \sqrt{3} / 2 z^{2}$.

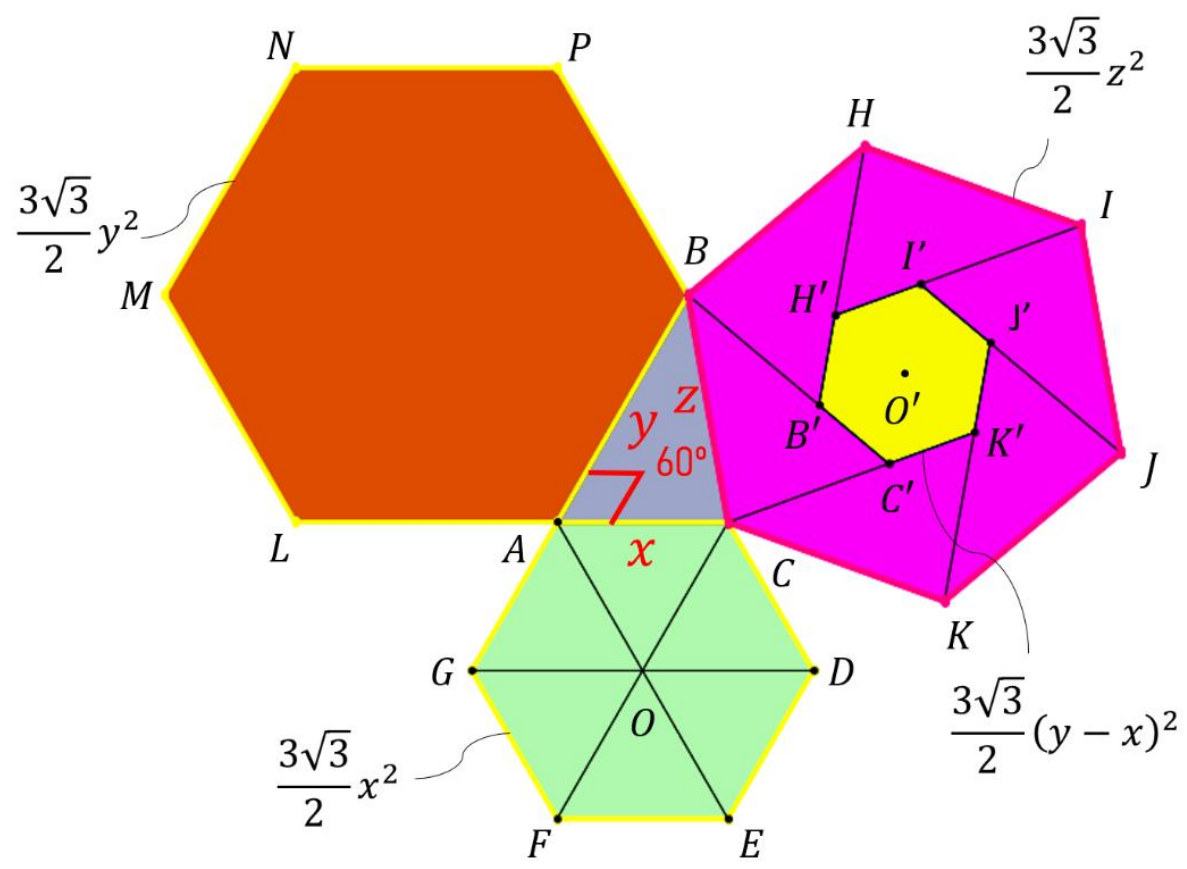

Figure 2. Forming hexagons on sides $x$ and $z$ of the extended Pythagorean theorem for the general case $x \neq y$

Turning to the left side of Figure 2, the internal division of hexagon ABPNML - such that it too integrates the central hexagon - is achieved via an unexpected step. Since the side $y=A B$ is larger than $z=C B$, the hexagon ABPNML is naturally larger than the hexagon CBHIJK. This allows for a copy of the hexagon CBHIJK to be translated and inscribed within the hexagon ABPNML, here termed hexagon C"B"H'I"J"K'. In other words, points C", B", H", I", $J$ " and K" become coincident with the edges of the hexagon ABPNML (where for instance point $H$ " is inline with edge NP), providing for a natural partition of hexagon ABPNML. This not only places a central hexagon $\mathrm{C} * \mathrm{~B} * \mathrm{H} * \mathrm{I} * \mathrm{~J} * \mathrm{~K} *$ within the regular hexagon ABPNML of side $y$ and area $3 \sqrt{3} / 2 y^{2}$, but it also defines a negative area $-3 \sqrt{3} / 2 x y$ composed of triangles B"B*H", H"H*I", I"I*J", J"J*K", K"K*C" and C"C*B". This negative area emerges 
mathematically from the minus term in $x^{2}-x y+y^{2}=z^{2}$, and geometrically it reduces the available area inside of the hexagon ABPNML such that it becomes $3 \sqrt{3} / 2 y^{2}-3 \sqrt{3} / 2 x y$. When this area is summed with the area $3 \sqrt{3} / 2 x^{2}$ of the hexagon ACDEFG side $x$, it gives the area $3 \sqrt{3} / 2 z$ of the hexagon CBHIJK side $z$, resulting in the equality

$$
\frac{3 \sqrt{3}}{2} y^{2}-\frac{3 \sqrt{3}}{2} x y+\frac{3 \sqrt{3}}{2} x^{2}=\frac{3 \sqrt{3}}{2} z^{2}
$$

This will be later proven geometrically and algebraically in sections 4.1 and 4.2 , respectively.

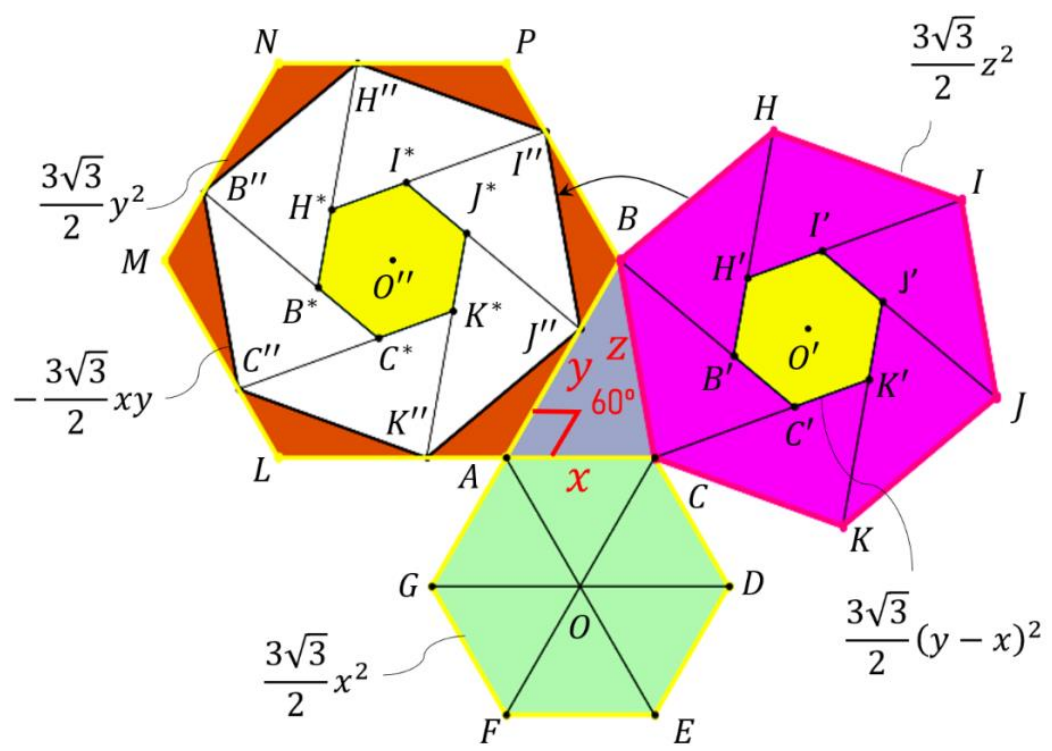

Figure 3. Forming a hexagon on side $y$ of the extended Pythagorean theorem for the general case $x \neq y$

\subsection{Particular Case $x=y$}

Now that the general case $x \neq y$ is explained, it is easier to understand how the particular case $x=y$ is formed. Imagine that the scalene triangle $\mathrm{ABC}$ of reference angle 60 degrees from Figure 3 becomes isosceles with two sides being equal $x=y$ (i.e., $\mathrm{AC}=\mathrm{AB}$ ), as shown in Figure 4. Then, not only does it become isosceles, but since the reference angle is 60 degrees, the scalene triangle $\mathrm{ABC}$ also becomes equilateral with $x=y=z$. While the hexagon of side $x$ in Figure 3 remains unchanged, the hexagon ABPNML associated with side $y$ and CBHIJK associated with side $z$ will change accordingly. As the triangle $\mathrm{ABC}$ becomes equilateral, its mirrored version located alongside $z=\mathrm{BC}$ - the triangle BCC' in Figure 3 - also becomes equilateral, with its inner point $\mathrm{C}^{\prime}$ tending to the centre point $\mathrm{O}^{\prime}$, becoming coincident in Figure 4. Likewise, all the six congruent revolving triangles - BCC', CKK', KJJ', JII', IHH' and $\mathrm{HBB}^{\prime}$ on the right of Figure 3 - will behave in the same manner, with all the points in the central hexagon B'H'I'J'K'C' tending to the central point O' - becoming BCO', CKO', KJO', JIO', IHO' and HBO' in Figure 4. In so doing, the central hexagon B'H'I'J'K' $C^{\prime}$ ' on the right vanishes, along with its area $3 \sqrt{3} / 2(y-x)^{2}$. Focusing now on the left side of Figure 4, since all sides of the ABC triangle become the same $x=y=z$, the hexagon ABPNML of side $y$ and area $3 \sqrt{3} / 2 y^{2}$ becomes equal to the hexagon ACDEFG of side $x$ and area $3 \sqrt{3} / 2 x^{2}$. The hexagon C"B"H"I"J"K" of side $z$ inscribed inside ABPNML of side $y$, will change as described above for hexagon CBHIJK associated with side $z$. That is, the central hexagon $\mathrm{B}^{*} \mathrm{H}^{*} \mathrm{I}^{*} \mathrm{~J} * \mathrm{~K}^{*} \mathrm{C}^{*}$ will also disappear, and the inscribed hexagon C"B" H"I"J"K" of negative area will become the same size as the hexagon ABPNML of side $y$, that is $-3 \sqrt{3} / 2 \mathrm{y}^{2}$. The overall balance of areas becomes clear - the positive area $3 \sqrt{3} / 2 y^{2}$ of the hexagon ABPNML cancels with the negative area $-3 \sqrt{3} / 2 y^{2}$ of the hexagon C"B"H"I"J"K" (now identical to ABPNML), while the remaining area comprising of the hexagon ACDEFG of area $3 \sqrt{3} / 2 x^{2}$ becomes the same as the hexagon CBHIJK of area $3 \sqrt{3} / 2 z^{2}$ in Figure 4 - resulting algebraically in

$$
\frac{3 \sqrt{3}}{2} y^{2}-\frac{3 \sqrt{3}}{2} y^{2}+\frac{3 \sqrt{3}}{2} x^{2}=\frac{3 \sqrt{3}}{2} z^{2}
$$

Which simplifies, as expected, to

$$
\frac{3 \sqrt{3}}{2} x^{2}=\frac{3 \sqrt{3}}{2}^{2}
$$




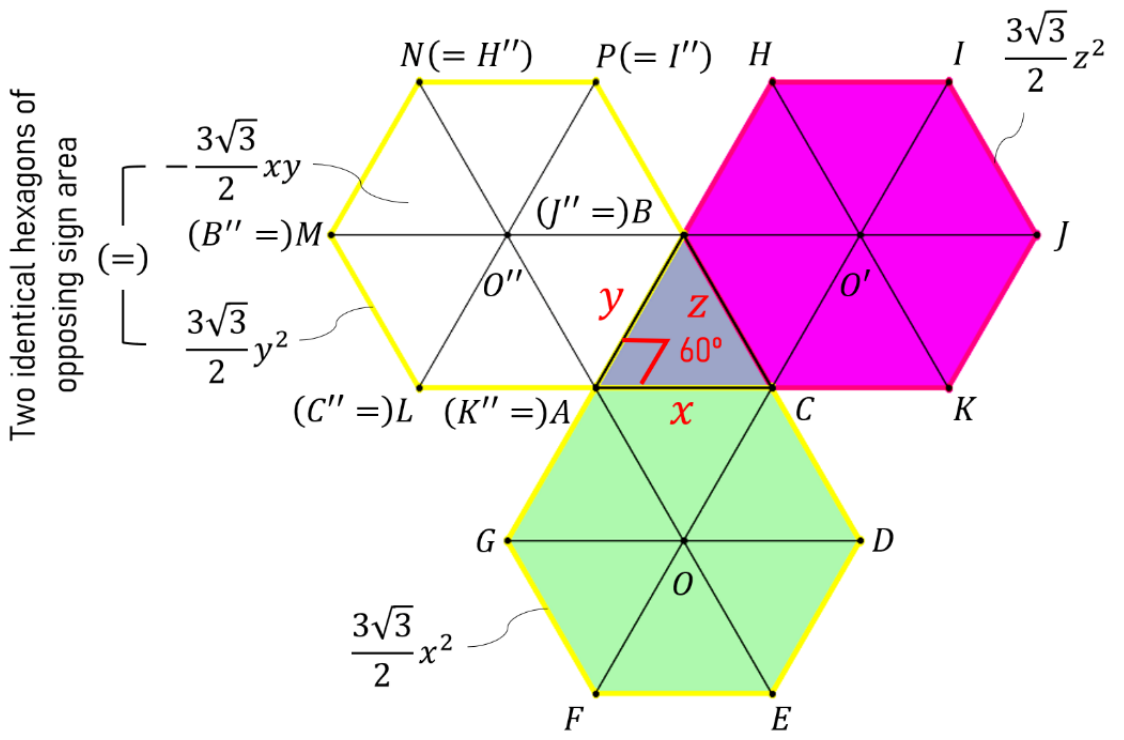

Figure 4. Extended (hexagon-based) Pythagorean theorem for the particular case $x=y$

\section{Formulating the Proof}

Theorem 1 (Extended Pythagorean theorem using Hexagons). If $x, y$ and $z$ are real numbers, then the lengths of the sides $x$ and $y$ of a 60-degree-angled triangle - forming the 60-degree angle between themselves - are related to the opposing side of the triangle $z$ via the sum of areas of the regular hexagons annexed to each corresponding side, minus a coupling area composed of six revolving 60-degree-angled triangles (themselves formed from the subtraction of an inscribed hexagon and a central hexagon), resulting in

\subsection{Geometric Part of the Proof}

$$
y^{2}-x y+x^{2}=z^{2}
$$

The geometric proof of the extended Pythagorean theorem using hexagons is shown in Figure 5. Considering the general case of $x \neq y$, the splitting of the area composed of the regular hexagons $3 \sqrt{3} / 2 x^{2}$ and $3 \sqrt{3} / 2 y^{2}$ minus the coupling combined area of six revolving regular triangles $-3 \sqrt{3} / 2 x y$ (Figure $5 \mathrm{a}$ ) will be shown to fit the totality of the area of the regular hexagon $3 \sqrt{3} / 2 z^{2}$ (Figure $5 b$ ). This is now explained.

Figure 5a shows the reference 60-degree-angled triangle $\mathrm{ABC}$ that replaces here the traditional right-angled triangle. Along the sides $x=\mathrm{AC}, \mathrm{y}=\mathrm{AB}$ and $\mathrm{z}=\mathrm{BC}$ of the 60 -degree-angled triangle $\mathrm{ABC}$, there are regular hexagons $\mathrm{ACDEFG}$ of area $3 \sqrt{3} / 2 x^{2}$, ABPNML of area $3 \sqrt{3} / 2 y^{2}$ and CBHIJK of area $3 \sqrt{3} / 2 z^{2}$, respectively, which replace the traditional squares $x^{2}, y^{2}$ and $z^{2}$ found in the classical theorem.

In the present 60 -degree-angled triangle $\mathrm{ABC}$, the side $y$ is larger than $z$, thus it is expected to find the hexagon CBHIJK of area $3 \sqrt{3} / 2 z^{2}$ inside the hexagon ABPNML of area $3 \sqrt{3} / 2 y^{2}$. Therefore, the first element to partition is the hexagon CBHIJK of side $z$ in Figure 5b, following by hexagons ACDEFG of side $x$ and ABPNML of side $y$ in Figure 5a. Mirroring the triangle $\mathrm{ABC}$ about side $\mathrm{BC}$ gives the triangle $\mathrm{BCC}^{\prime}$ in Figure $5 \mathrm{~b}$, and revolving it six times around the center O' gives congruent triangles BCC', CKK', KJJ', JII', IHH' and HBB'. As a result, the inner side of these triangles form the central hexagon B'H'I'J'K' $C^{\prime}$ of side $y-x$ (e.g., B'C') and area $3 \sqrt{3} / 2(y-x)^{2}$, while the outer side forms the hexagon CBHIJK of side $\mathrm{z}$ (e.g., BC) and area $3 \sqrt{3} / 2 z^{2}$.

Now returning back to the bottom of Figure 5a, the hexagon ACDEFG of side $x$ is split into six equilateral triangles AOC, COD, DOE, EOF, FOG and GOA. The analysis proceeds to identify the partition of the hexagon ABPNML of side $y$ and area $3 \sqrt{3} / 2 y^{2}$. Inscribing a copy of the partition of the hexagon CBHIJK of side $z$ (Figure $5 \mathrm{~b}$ ) into the hexagon ABPNML of side $y$ to the left of Figure 5a, further divides the later into a central hexagon $\mathrm{B} * \mathrm{H}^{*} \mathrm{I}^{*} \mathrm{~J} * \mathrm{~K} * \mathrm{C} *$ of area $3 \sqrt{3} / 2(y-x)^{2}$, six revolving 60-degree-angled triangles - B"B*H", H"H*I", I"I*J", J"J*K", K"K*C" and $\mathrm{C}$ " $\mathrm{C} * \mathrm{~B}$ " - and finally the difference between hexagon CBHIJK side $z$ and ABPNML side $y$, with an additional six triangles - B"NH", H"PI", I"BJ", J"AK", K"LC" and C"MB" - each of area $\sqrt{3} / 4 x(y-x)$. Take for example the triangle $\mathrm{J}$ " $\mathrm{AK}$ " located along the side $x=\mathrm{AB}$. A circular arc segment centered at point $\mathrm{A}$ and starting at point $\mathrm{C}$ is drawn (counterclockwise), encountering point $\mathrm{J}$ ", which shows that the segment $\mathrm{J}$ "A has a length $x$. Since the hexagon ABPNML has a side $y$, then the other segment AK" of the triangle J"AK" has length $y-x$. 


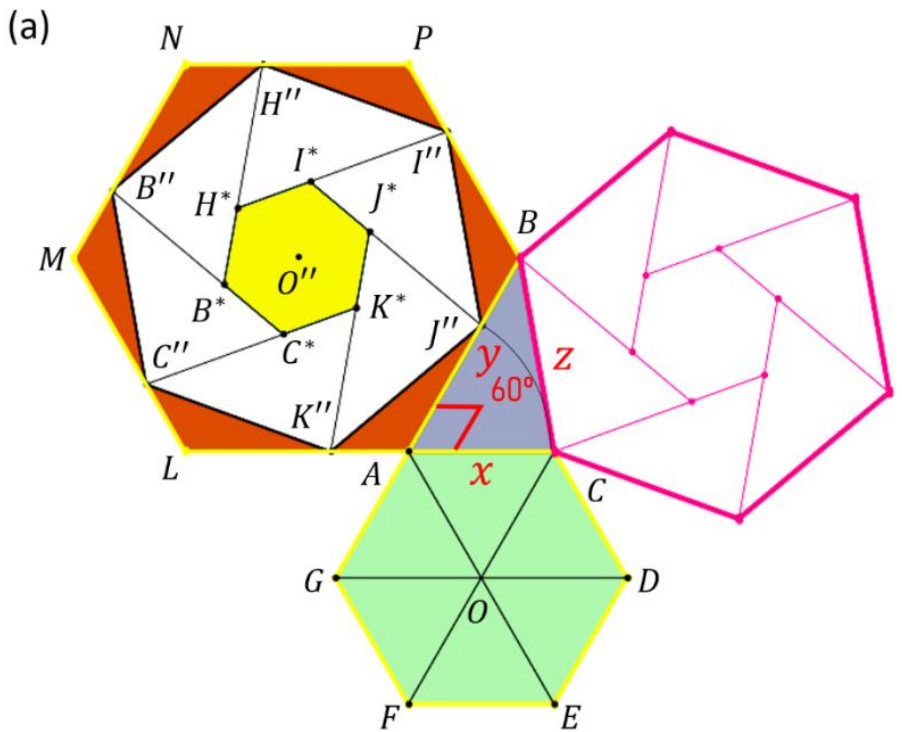

(b)

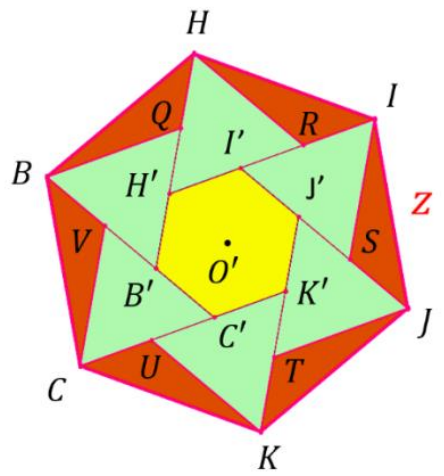

Figure 5. Geometric proof of the extended Pythagorean theorem relating hexagonal areas (a) $3 \sqrt{3} / 2 x^{2}, 3 \sqrt{3} / 2 y^{2}$

$$
\text { minus } 3 \sqrt{3} / 2 x y \text { to (b) } 3 \sqrt{3} / 2 z^{2}
$$

Now that the partitioning of the hexagons is complete, it is possible to verify the conservation of area described by the equation $x^{2}-x y+y^{2}=z^{2}$ governing the extended Pythagorean theorem using hexagons. The six equilateral triangles - AOC, COD, DOE, EOF, FOG and GOA - that compose the hexagon ACDEFG of side $x$ (in Figure 5a) appear in Figure $5 \mathrm{~b}$ inside the hexagon CBHIJK of side $z$ - triangles BB' $\mathrm{Q}, \mathrm{HH}$ 'R, II'S, JJ'T, KK'U and CC'V. The hexagon ABPNML of side $y$ in Figure 5a is split into three groups: first, six triangles B"B*H", H"H*I", I"I*J", $J$ "J*K", K"K*C" and C"C*B" that form a negative area cancel part of the positive area of the hexagon ABPNML (with which it overlaps). Second, the inner edges of these triangles form a central hexagon $B * H^{*} I^{*} J * K^{*} C^{*}$ in Figure 5a, which is also found in Figure 5b at the core of the hexagon CBHIJK of side $z$, as the central hexagon B'H'I'J'K'C'. And thirdly, the difference in size between the hexagon ABPNML of side $y$ and the inscribed hexagon CBHIJK of side $z$ within it, formed in Figure 5a six outer triangles B"NH", H"PI", I"BJ", J"AK", K"LC" and C"MB". These are found again in Figure 5b as BQH, HRI, ISJ, JTK, KUC and CVB, thus completing the hexagon CBHIJK of side $z$. It was shown that in Figure 5a, B"N $=x$ and $\mathrm{NH}^{\prime \prime}=y-x$ for triangle B"NH" (previously defined with the example of triangle J"AK") in Figure 5a, are the same sides as BQ and HQ for the triangle BQH in Figure 5b. In fact, the triangle $\mathrm{B}$ "NH" in Figure 5a is the inverted version (along B" $\mathrm{H}$ ") of triangle BQH in Figure 5b. It is straightforward to see that if $\mathrm{B}^{\prime} \mathrm{H}$ in triangle $\mathrm{BB} \mathrm{H}^{\prime} \mathrm{H}$ of Figure $5 \mathrm{~b}$ (same as $\mathrm{ABC}$ in Figure $5 \mathrm{a}$ ) is $y$, then the length $\mathrm{HQ}$ in Figure $5 \mathrm{~b}$ is the difference of $\mathrm{B}^{\prime} \mathrm{H}=y$ and $\mathrm{B}^{\prime} \mathrm{Q}=x$, giving $\mathrm{HQ}=y-x$. Therefore, triangle $\mathrm{B}$ " $\mathrm{NH}^{\prime}$ ' in Figure $5 \mathrm{a}$ is the same as $\mathrm{BQH}$ in Figure $5 \mathrm{~b}$. It is an overall conclusion from the above that the positive area found inside the hexagon ABPNML of side $y$ plus the area inside the hexagon ACDEFG of side $x$ (both in Figure 5a) equates to the area found inside the hexagon CBHIJK of side $z$ (in Figure 5b). This completes the geometric part of the proof.

\subsection{Algebraic Part of the Proof}

The algebraic process of adding areas, leading to the extended version of the Pythagorean theorem of equation $x^{2}-x y+y^{2}=z^{2}$, follows from the aforementioned geometric description in section 4.1. The final equation is reached via the formulation of two individual equations: one emerges when analyzing the left part of the theorem that pertains to the hexagons of side $x$ and $y$ (Figure 5a), and the second when analyzing the right part of the theorem that pertains to the hexagon of side $z$ (Figure 5b). Both Figure 5a and 5b represent (in terms of added areas) each an equation, and both incorporate a central hexagon that links both equations, providing the desire end solution. Figure 5a shows a positive area inside the hexagon ABPNML (associated with side $y=A B$ ) comprising of $3 \sqrt{3} / 2 y^{2}$ minus the negative area $-3 \sqrt{3} / 2 x y$ resulting from the six congruent revolving triangles equal to $\mathrm{ABC}$, that is $\mathrm{B}$ " $\mathrm{B} * \mathrm{H}$ ", $\mathrm{H}^{\prime} \mathrm{H}^{*} \mathrm{I}$ ", I"I*J", J"J*K", K" $\mathrm{K} \mathrm{C}^{*}$ " and $\mathrm{C}$ "C*B". This positive area is equal to the sum of the central hexagon $B * H^{*} I * J * K^{*} C^{*}$ of area $3 \sqrt{3} / 2(y-x)^{2}$ plus the area of the six revolving smaller triangles $-\mathrm{B}$ "NH", H"PI", I"BJ", J"AK", K"LC" and C"MB" - comprising of an overall area $6 \times \sqrt{3} / 4 x(y-x)$, resulting in

$$
6 \frac{\sqrt{3}}{4} x(y-x)+\frac{3 \sqrt{3}}{2}(y-x)^{2}=\frac{3 \sqrt{3}}{2} y^{2}-6 \frac{\sqrt{3}}{4} x y
$$


Re-arranging the equation gives

$$
6 \frac{\sqrt{3}}{4} x y+6 \frac{\sqrt{3}}{4} x(y-x)+\frac{3 \sqrt{3}}{2}(y-x)^{2}=\frac{3 \sqrt{3}}{2} y^{2}
$$

This forms one of the equations. The second equation is retrieved from Figure $5 \mathrm{~b}$, and is described as follows. The hexagon CBHIJK in Figure $5 b$ of area $3 \sqrt{3} / 2 z^{2}$ is composed of six revolving equilateral triangles - BQB', HRH', ISI', JTJ', KUK' and CVC' - that have a combined area $3 \sqrt{3} / 2 x^{2}$ (same as the hexagon ACDEFG), plus six smaller triangles $-\mathrm{BQH}$, HRI, ISJ, JTK, KUC and CVB - of area $\sqrt{3} / 4 x(y-x)$ each, being complete with a central hexagon B'H'I'J'K'C' of area $3 \sqrt{3} / 2(y-x)^{2}$, giving

$$
\frac{3 \sqrt{3}}{2} x^{2}+6 \frac{\sqrt{3}}{4} x(y-x)+\frac{3 \sqrt{3}}{2}(y-x)^{2}=\frac{3 \sqrt{3}}{2} z^{2}
$$

Subtracting Eq.(4) from Eq.(5) removes the linking term - the central hexagon $3 \sqrt{3} / 2(y-x)^{2}-$ and Eq.(5) becomes

$$
\frac{3 \sqrt{3}}{2} x^{2}-6 \frac{\sqrt{3}}{4} x y=\frac{3 \sqrt{3}}{2} z^{2}-\frac{3 \sqrt{3}}{2} y^{2}
$$

Expanding and simplifying the terms on the left, and placing the $3 \sqrt{3} / 2 y^{2}$ on the other side gives

$$
\frac{3 \sqrt{3}}{2} x^{2}-\frac{3 \sqrt{3}}{2} x y+\frac{3 \sqrt{3}}{2} y^{2}=\frac{3 \sqrt{3}}{2}^{2}
$$

Conveniently removing the coefficient $3 \sqrt{3} / 2$ results in the required relation

$$
x^{2}-x y+y^{2}=z^{2}
$$

This completes the algebraic part of the proof, and thus the proof overall.

\section{References}

Canadian Ministry of Education. (2020). The Ontario Curriculum, Grades 1-8: Mathematics. Retrieved from https://www.dcp.edu.gov.on.ca/en/curriculum/elementary-mathematics

Curtis, H. D. (2010). Orbital Mechanics for Engineering Students, (2nd ed.). Butterworth-Heinemann. https://doi.org/10.1016/C2009-0-19374-1

Euclid, Heath, T. L., \& Heiberg, J. L. (1908). The thirteen books of Euclid's Elements. Cambridge, The University Press (pp. 405-408). Retrieved from https://archive.org/details/thirteenbookseu02heibgoog/mode/2up

Maor, E. (2007). The Pythagorean Theorem: A 4,000 Year History. Princeton University Press. https://doi.org/10.2307/j.ctvh9w0ks

Mark, H., \& Workman, Jr. J. (2018). Chemometrics in Spectroscopy. (2nd ed.). Academic Press. https://doi.org/10.1016/C2015-0-04023-0

Parisher, R. A., \& Rhea, R. A. (2012). Pipe Drafting and Design. (3rd ed.). Gulf Professional Publishing https://doi.org/10.1016/C2011-0-06090-8

Pickover, C. A. (2012). The Math Book: From Pythagoras to the 57th Dimension, 250 Milestones in the History of Mathematics. Sterling Milestones.

Rawlins, J. C. (2000). Basic AC Circuits (2nd ed.). Newnes Publishing. https://doi.org/10.1016/B978-0-7506-7173-6.X5000-7

Teia, L. (2015). Pythagoras' triples explained via central squares. Australian Senior Mathematics Journal, 29(1), 7-15.

\section{Copyrights}

Copyright for this article is retained by the author(s), with first publication rights granted to the journal.

This is an open-access article distributed under the terms and conditions of the Creative Commons Attribution license (http://creativecommons.org/licenses/by/4.0/). 